Case report

\title{
Granular dot-like staining with MLH1 immunohistochemistry is a clone- dependent artefact
}

\author{
S. Dasgupta ${ }^{a}, *$, P.C. Ewing-Graham ${ }^{a}$, F.H. Groenendijk ${ }^{a}$, O. Stam ${ }^{b}$, K.E. Biermann ${ }^{a}$, M. Doukas ${ }^{a}$, \\ H.J. Dubbink ${ }^{a}$, M.F. van Velthuysen ${ }^{a}$, W.N.M. Dinjens ${ }^{\mathrm{a}}$, M.R. Van Bockstal ${ }^{\mathrm{a}, \mathrm{c}}$ \\ ${ }^{a}$ Department of Pathology, Erasmus MC, University Medical Centre Rotterdam, the Netherlands \\ ${ }^{\mathrm{b}}$ Department of Pathology, Pathan BV, Sint Franciscus Gasthuis, Rotterdam, the Netherlands \\ ${ }^{\mathrm{c}}$ Department of Pathology, University Clinics Saint-Luc, Brussels, Belgium
}

\section{A R T I C L E I N F O}

\section{Keywords:}

DNA mismatch repair

Microsatellite instability

MLH1

PMS2

Immunohistochemistry

Dot-like staining

\begin{abstract}
A B S T R A C T
Immunohistochemistry (IHC) for DNA mismatch repair proteins MLH1, PMS2, MSH2, and MSH6 is used for microsatellite instability (MSI) screening in colorectal carcinoma (CRC) and endometrial carcinoma (EC). Loss of PMS2, with retained MLH1 staining occurs in germline mutations of PMS2 gene, and is an indication for genetic testing. We report a pitfall of immunohistochemical interpretation in an EC, initially regarded as MLH1-positive and PMS2-negative. Review of the MLH1-IHC (M1-clone) revealed a granular, dot-like, nuclear staining. On repeating the MLH1-IHC with a different clone (ES05-clone), complete negativity was noted, and on molecular testing, MLH1 promotor methylation was detected. The dot-like pattern was therefore adjudged a clone-dependent artefact. On reviewing the archived MLH1-IHC slides, we observed the same dot-like pattern in two CRCs; in both cases the M1-clone had been used. Awareness of this artefact may prevent reporting errors, and unnecessary referrals for germline mutation testing.
\end{abstract}

\section{Introduction}

The DNA mismatch repair (MMR) complex comprises the proteins MLH1, MSH2, MSH6, and PMS2, which exist as functional heterodimers MLH1/PMS2 and MSH2/MSH6 [1]. Inactivation of the MMR genes allows the accumulation of replication errors within the short nucleotide repeat sequences known as microsatellites, thereby giving rise to microsatellite instability (MSI) [1,2].

MSI resulting from heterozygous germline mutations of the MMR genes causes Lynch syndrome (LS), which predisposes affected individuals to cancers of several sites, most notably, colorectal carcinoma (CRC) and endometrial carcinoma (EC) [3]. On the other hand, MLH1 promoter inactivation by hyper-methylation at specific $\mathrm{CpG}$ sites leads to sporadic MSI, which can also be associated with CRCs and ECs [4].

MMR deficient ECs and CRCs occurring in LS are known to have a better prognosis and response to chemotherapy than MMR proficient ECs and CRCs [5]. Detection of LS also enables counselling and surveillance for patients and their family members [2]. Universal testing for MSI of newly diagnosed CRCs and ECs has therefore been recommended [6,7].

The gold-standard for MSI detection is polymerase chain reaction (PCR)-based amplification of the specific microsatellite repeats, and immunohistochemistry (IHC) for the MMR proteins offers an efficient yet economical screening tool for MSI [2,7]. On IHC, loss of both MLH1 and PMS2 staining is seen if MLH1 gene is inactivated, whereas loss of both MSH2 and MSH6 staining is seen in case of inactivation of MSH2 gene [8]. This is because PMS2 and MSH6 proteins cannot exist stably in the absence of their respective obligatory partners, MLH1 and MSH2. Inactivation of PMS2 and MSH6 genes however, lead to isolated loss of PMS2 and MSH6 staining respectively [8]. For MLH1/PMS2-deficient tumours, recent guidelines mandate $M L H 1$ promoter methylation or $B R A F$ exon 15 mutation analysis, to exclude sporadic MSI [2].

The MMR-IHC thus helps us determine the subsequent investigations [2]. Accurate interpretation of MLH1-IHC is crucial to avoid misdiagnoses of isolated PMS2 deficiency, and inappropriate mutation analysis. Here, we report a clone-dependent artefactual staining pattern of MLH1, which is a pitfall of immunohistochemical interpretation.

\footnotetext{
*Corresponding author at: Department of Pathology, Erasmus MC, University Medical Centre Rotterdam, Postbus 2040, BE-gebouw, 3000, CA Rotterdam, the Netherlands.

E-mail addresses: s.dasgupta@erasmusmc.nl (S. Dasgupta), p.ewing@erasmusmc.nl (P.C. Ewing-Graham), f.groenendijk@erasmusmc.nl (F.H. Groenendijk), o.stam@pathan.nl (O.Stam), k.biermann@erasmusmc.nl (K.E. Biermann), m.doukas@erasmusmc.nl (M. Doukas), h.dubbink@erasmusmc.nl (H.J. Dubbink), m.vanvelthuysen@erasmusmc.nl (M.F. van Velthuysen), w.dinjens@erasmusmc.nl (W.N.M. Dinjens), mieke.vanbockstal@uclouvain.be (M.R. Van Bockstal).
} 


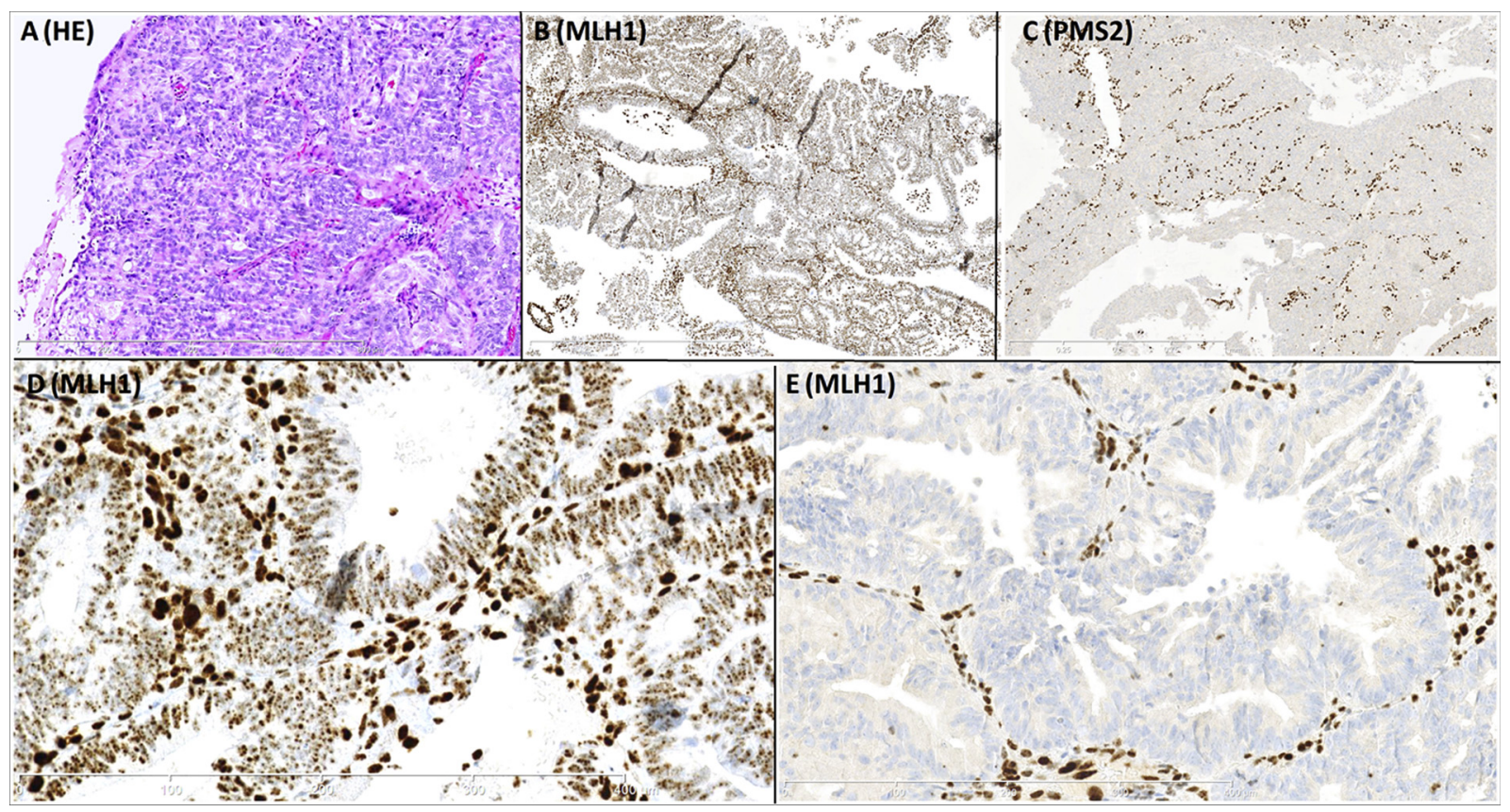

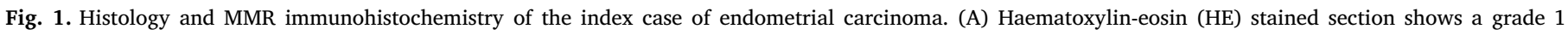

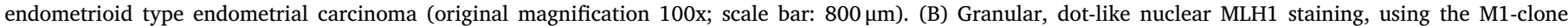

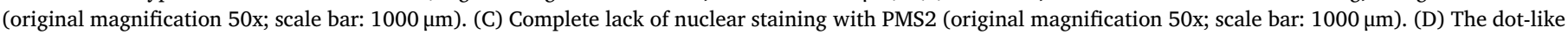

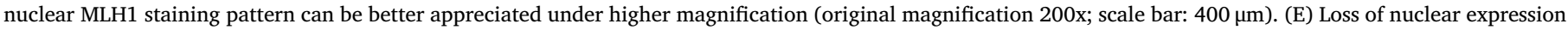
of MLH1 on repeating MLH1 immunohistochemistry with the ES05-clone (original magnification 200x; scale bar: $400 \mu \mathrm{m}$ ).

\section{Materials and methods}

\subsection{Index case}

An endometrial biopsy, diagnosed as endometrioid EC (grade 1), originally reported as isolated loss of PMS2 staining, was sent to the department of Pathology, Erasmus MC, for a second opinion regarding the MMR-IHC. MLH1-IHC had been originally performed with the M1clone, ready-to-use mouse monoclonal antibody (Ventana Medical Systems, Roche Diagnostics, Mannheim, Germany).

On reviewing the MLH1-IHC slide, a granular, dot-like staining pattern in the nuclei of the tumor cells was noted. We repeated MLH1IHC using the ES05-clone (NCL-L-MLH1, Leica Biosystems Newcastle, Newcastle Upon Tyne, UK). The tumour showed no MLH1 expression (Fig. 1). For confirmation of the MSI-status, Pentaplex PCR with a panel comprising NR-21, BAT-25, BAT-26, NR-24 and Mono-27 was performed. To determine whether the MSI was sporadic, $M L H 1$ promoter methylation analysis was performed by Methylation-Specific Multiplex Ligation-dependent Probe Amplification (MS-MLPA, MRC Holland, ME011 kit), using normal reference DNA as control (Fig. 2).

\subsection{Review of archived MMR IHC slides}

The index case prompted us to review the MMR-IHC slides from the departmental archive, to investigate the frequency of this dot-like staining, as the M1-clone was previously in use in our Pathology laboratory.

Between January 2014 and February 2015, the M1-clone was in use. We reviewed MLH1 and PMS2 slides of cases reported as MLH1-negative in this period. Since March 2015, our laboratory uses the ES05clone. For this clone, we reviewed the MLH1 and PMS2 slides of cases reported as MLH1-negative between March 2015 and February 2016. In addition, for both clones, we reviewed a set of randomly selected MLH1-positive cases, to detect any false-positives. Staining protocols for both clones are detailed in Table 1.

\section{Results}

\subsection{Index case}

The granular, dot-like staining with MLH1-IHC in the nuclei of the tumor cells was clearly distinct from the homogenous staining in the internal control, i.e. nuclei of the lymphocytes and fibroblasts. This pattern was not noted with the ES05-clone. In this case, the nuclei of tumour cells were completely negative, whereas the nuclei of lymphocytes and fibroblasts showed strong, homogenous staining (Fig. 1).

In view of the lack of staining with both MLH1 and PMS2 on repeat IHC, the index case was diagnosed as harbouring an MSI. This was confirmed with the detection of an MSI-phenotype on Pentaplex PCR. MS-MLPA analysis showed hypermethylation of the MLH1 promotor region in the tumor cells, indicating a sporadic MSI, and ruling out LS (Fig. 2).

The granular, dot-like staining pattern was therefore adjudged an M1-clone-dependent artefact.

\subsection{Review of archived MLH1 slides}

M1-clone: Forty-eight cases had been reported as MLH1-negative, which comprised 41 CRCs, 5 ECs, 1 ovarian carcinoma, and 1 duodenal adenocarcinoma. The slides could be retrieved and reviewed for $79 \%$ (38/48) of these (Table 2). In 2 CRCs, the same granular, dot-like nuclear staining pattern of MLH1 was noted; PMS2 was negative in both (Fig. 3). Both cases showed an MSI phenotype on Pentaplex PCR, and detection of MLH1 promotor hypermethylation on MS-MLPA analysis indicated a sporadic MSI.

The remaining cases were completely negative for both MLH1 and PMS2.

ES05-clone: Forty-nine cases had been reported as MLH1-negative, comprising 45 CRCs, 2 ECs, 1 ovarian carcinoma, and 1 breast carcinoma. The slides could be retrieved and reviewed for $69 \%(34 / 49)$, and all of these were completely negative for both MLH1 and PMS2 (Table 2). 


\begin{tabular}{|l|l|}
\hline MS-MLPA Analysis Report - SoftGenetics \\
\hline Software: GeneMarker V2.4.0 & Analysis Type: Promoter Methylation \\
\hline Project: Untatled & Compare Type: MLPA Ratio \\
\hline Technician: & Mormalization By: Population Normalizaton \\
\hline Report Time: 05/17/2019 - 13:44:49 & Quantification By: Peak Height \\
\hline Panel: MS-MLPA ME011-83 & Classification: Normal $<=0.30<$ Methylated \\
\hline Control: M18-2081T2_L_A01_008.fsa & Report Value Type: Peak Ratio \\
\hline Auto Slope Correction: $1.28+-2.5 \mathrm{e}-03 \mathrm{x}$ & \\
\hline
\end{tabular}

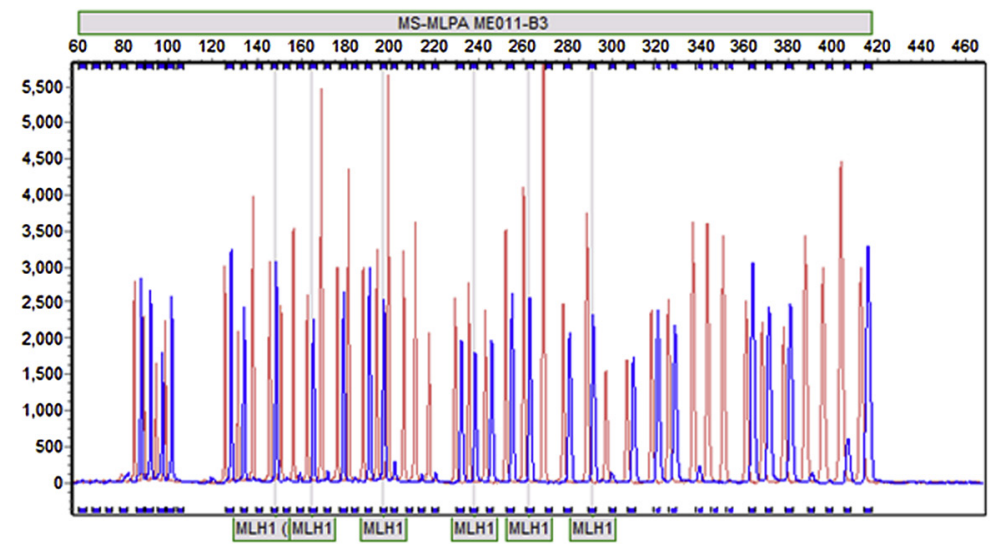

Machine: $3730 \_$PA-21375-003

Run Time: 10/11/2018 - 12:24:18 -> 10/11/2018 - 13:08:30

\begin{tabular}{|l|l|l|}
\hline Conclusion & \multicolumn{2}{|l|}{} \\
\hline & Date & Initial \\
\hline Authorization 1 & & \\
\hline Authorization 2 & & \\
\hline
\end{tabular}
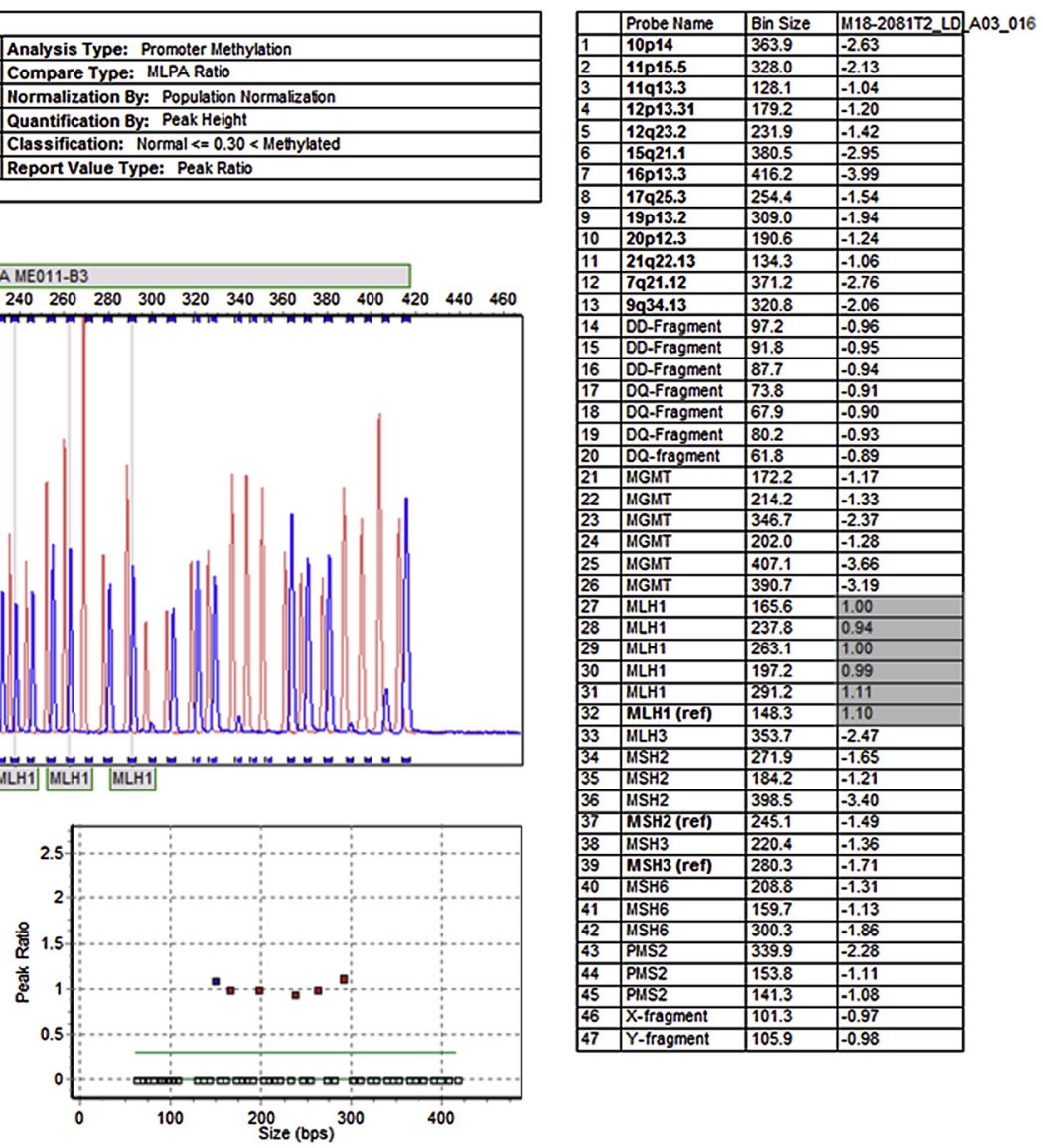

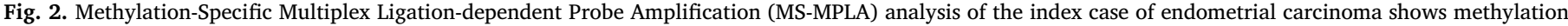
peaks, indicating the presence of a methylated $M L H 1$ promotor region.

Table 1

Staining protocol followed for MLH1 immunohistochemistry.

\begin{tabular}{|c|c|c|c|c|c|}
\hline Clone & Dilution & Manufacturer & $\begin{array}{l}\text { Heat induced antigen } \\
\text { retrieval }\end{array}$ & Incubation & Visualation kit \\
\hline M1 & Ready to use & 790-4535/Ventana-Roche & $\mathrm{CC} 1,32$ minutes at $97^{\circ} \mathrm{C}$ & 16 minutes at $36^{\circ} \mathrm{C}$ & Optiview DAB KIT \\
\hline ES05 & $1: 75$ & $\begin{array}{l}\text { NCL-L-MLH-1/ Leica- } \\
\text { Novocastra }\end{array}$ & $\mathrm{CC} 1,48$ minutes at $100^{\circ} \mathrm{C}$ & $\begin{array}{l}60 \text { minutes at } 37^{\circ} \mathrm{C} \text {, followed by Optiview amplification for } 4 \\
\text { minutes }\end{array}$ & Optiview DAB KIT \\
\hline
\end{tabular}

Table 2

Review of the archived MLH-1 immunohistochemistry (IHC) slides reported as negative, and the corresponding PMS2 IHC slides.

\begin{tabular}{|c|c|c|c|c|c|}
\hline \multirow[t]{2}{*}{ Clone (n) } & \multirow[t]{2}{*}{ Lesions (n) } & \multicolumn{2}{|c|}{ Dot-like pattern with MLH1-IHC } & \multicolumn{2}{|c|}{ PMS2-IHC } \\
\hline & & Present & Absent & Present & Absent \\
\hline \multirow[t]{4}{*}{ M1 (38) } & Colorectal carcinoma (32) & 2 & 30 & - & 32 \\
\hline & Endometrial carcinoma (4) & - & 4 & - & 4 \\
\hline & Duodenal adenocarcinoma (1) & - & 1 & - & 1 \\
\hline & Ovarian carcinoma (1) & - & 1 & - & 1 \\
\hline \multirow[t]{3}{*}{ ES05 (34) } & Colorectal carcinoma (32) & - & 32 & - & 32 \\
\hline & Endometrial carcinoma (1) & - & 1 & - & 1 \\
\hline & Ovarian carcinoma (1) & - & 1 & - & 1 \\
\hline
\end{tabular}

None of the randomly selected MLH1-positive tumours (M1-clone: $\mathrm{n}=157$, ES05-clone: $\mathrm{n}=157$ ) showed the similar dot-like pattern. All of these showed strong, homogeneous nuclear staining for both MLH1 and PMS2.

\section{Discussion}

The granular dot-like aberrant staining pattern with MLH1-IHC may be misinterpreted as positive staining, particularly under low magnification. Negative staining with PMS2 in such cases can prompt a 


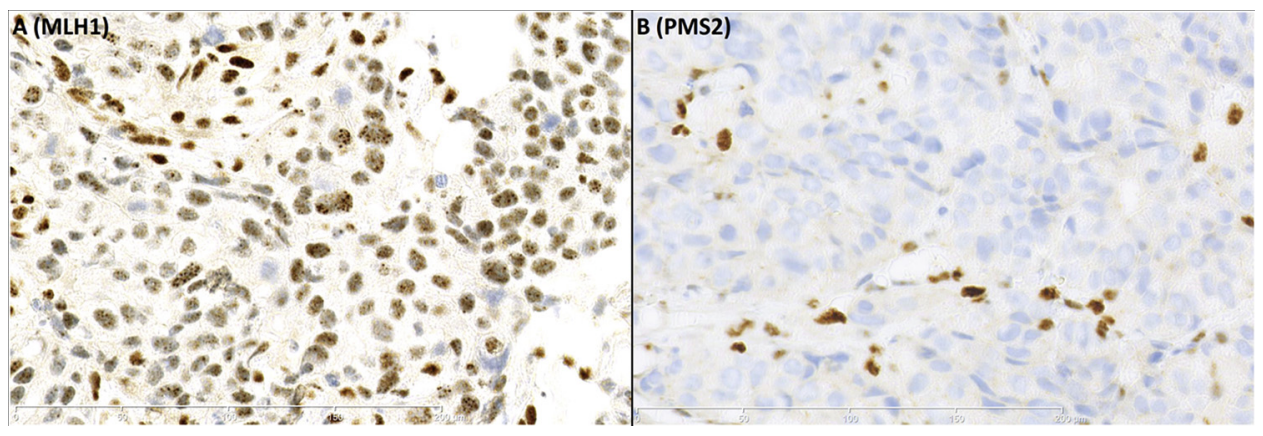

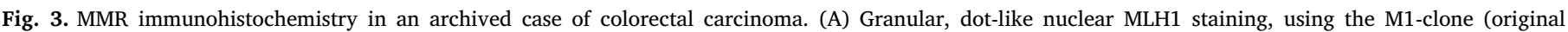
magnification $400 x$, scale bar is $200 \mu \mathrm{m}$ ). (B) Complete lack of nuclear staining with PMS2 (original magnification $400 x$, scale bar is $200 \mu \mathrm{m}$ ).

germline mutation analysis for PMS2 gene.

MMR-IHC should be interpreted as positive only when there is an unequivocally strong nuclear immunoreactivity, with comparable staining in the internal controls [2]. Loss of MMR protein expression is characterised by complete absence of staining of one or more MMR proteins in the nuclei of the tumor cells. A gradient of expression in the normal crypts, which fades out towards the lumen should be present, along with strong staining in the stromal fibroblasts and lymphocytes [2]. Loss of nuclear expression of MMR protein with variable cytoplasmic staining, or patchy nuclear expression with strong staining in the internal controls, should also be interpreted as loss of MMR protein expression [2].

For MLH1-IHC, Markow et al. described a nucleolar staining pattern as a potential pitfall [9]. We noted a similar MLH1 staining pattern, which we described as granular, dot-like, in 3 cases from our series, and all had been stained with the M1-clone. This aberrant staining was described in the Nordic Immunohistochemical Quality Control (NordiQC) report, for the M1-clone (Ventana) and the G168-728-clone (Cell Marquee) [10]. This assessment run ascribed $67 \%$ of the insufficient MLH1-IHC to false-positive nuclear staining, or cytoplasmic/background staining interfering with interpretation [10]. As per this report, ES05-clone provided the most sufficient results for MLH1-IHC [10].

Niu et al. recently reported a heterogeneous punctate nuclear staining with MLH1-IHC in $6 \mathrm{ECs}$, which had been originally reported as isolated PMS2-negative [11]. M1-clone had been used for all of these cases. Similar to our cases, all of them showed complete negativity on repeating MLH1-IHC with ES05-clone, and MLH1 promotor was methylated [11]. Loughrey et al. reported a similar aberrant MLH1-IHC pattern with the M1-clone, in 9 of the 16 PMS2-negative CRCs from his cohort, and 4 of these showed somatic BRAF V600E mutation [12]. Loughrey et al. therefore suggested that the punctate pattern represents a non-functional MLH1 protein variant with retained antigenicity, generated due to the somatic mutation [12]. For ECs, Watkins et al. reported an association of subclonal loss of MLH1 staining (Novocastra, clone NCL-L-MLH1) with MLH1 promotor methylation [13]. Subclonal loss was defined as abrupt and complete regional loss of MMR protein expression with intervening stromal positivity [13]. These associations deserve further exploration through larger studies.

Nucleolar pattern of staining has also been observed with MSH6IHC, in tumors post-neoadjuvant chemo/radiotherapy, using the 44/ BD-clone (BD Transduction Laboratories, Franklin Lakes, NJ, USA) [14]. In these cases, tumor hypoxia or oxidative stress has been implicated to give rise to this aberrant staining [15]. Other aberrant patterns, e.g. peri-nuclear staining, and extremely patchy staining, has also been reported for MMR-IHC, and has been majorly ascribed to technical problems, such as time and duration of fixation, or decalcification [2]. We hypothesize that addition of amplification steps, and prolongation of the antigen retrieval may increase the occurrence of nuclear dots.

MMR-IHC is a simple and cost-effective tool for MSI screening, with a high sensitivity and specificity [2]. Awareness amongst pathologists of the IHC pitfalls, such as the one we present here, will ensure optimal utilisation of this tool, and prevent misdirected genetic investigations. Moreover, the use of a monoclonal anti-MLH1 antibody, which has been consistently reported as robust can prevent false-positive IHC results. Even in a large series, artefactual staining patterns are detected in only a minority of cases; this emphasises the need for regular periodic external proficiency testing.

\section{Conclusion}

Granular dot-like MLH1 staining pattern represents an M1-clone dependent artefact, and should not be misdiagnosed as isolated PMS2negative.

\section{Author contribution statement}

All the authors provided valuable scientific input for the study, and revised the manuscript critically for important intellectual content. All the authors gave their approval for the version to be published and agree to be accountable for all of the aspects of the study.

\section{Compliance with ethical standards}

The Dutch national guidelines state that no ethical approval is required for the use of anonymous leftover tissue (www.federa.org) and this is also part of a standard treatment agreement with patients at Erasmus MC.

\section{Declaration of Competing Interest}

The authors have no conflicts of interest. No external funding was received for this study. All the authors gave their approval for the version to be published and agree to be accountable for all of the aspects of the study.

\section{Acknowledgements}

The authors would like to thank Ms. Erina Vonk Noordegraaf-de Leeuw, Ms. Sharmiela Ramlal, Mr. Hans Stoop, Ms. Peggy Atmodimedjo, Mr. Hans de Bruijn, and Mr. Frank van der Panne for their excellent technical assistance.

\section{References}

[1] J. Shia, N.A. Ellis, D.S. Klimstra, The utility of immunohistochemical detection of DNA mismatch repair gene proteins, Virchows Arch. 445 (2004) 431-441, https:// doi.org/10.1007/s00428-004-1090-5.

[2] A. Remo, M. Fassan, G. Lanza on behalf of AIFEG and GIPAD, Immunohistochemical evaluation of mismatch repair proteins in colorectal carcinoma: the AIFEG/GIPAD proposal, Pathologica 108 (2016) 104-109.

[3] B. Djordjevic, R.R. Broaddus, Role of the clinical pathology laboratory in the 
evaluation of endometrial carcinomas for Lynch syndrome, Semin. Diagn. Pathol 31 (2014) 195-204, https://doi.org/10.1053/j.semdp.2014.03.005.

[4] J.M. Cunningham, E.R. Christensen, D.J. Tester, et al., Hypermethylation of the hMLH1 promoter in colon cancer with microsatellite instability, Cancer Res. 58 (1998) 3455-3460.

[5] D. Klingbiel, Z. Saridaki, A.D. Roth, F.T. Bosman, M. Delorenzi, S. Tejpar, Prognosis of stage II and III colon cancer treated with adjuvant 5-fluorouracil or FOLFIRI in relation to microsatellite status: results of the PETACC-3 trial, Ann. Oncol. 26 (2015) 126-132, https://doi.org/10.1093/annonc/mdu499 Epub 2014 Oct 30.

[6] E.J. Crosbie, N.A.J. Ryan, M.J. Arends, et al., The Manchester International Consensus Group Recommendations for the Management of Gynecological Cancers in Lynch Syndrome, Genet. Med. (2019), https://doi.org/10.1038/s41436-019. 0489-y [Epub ahead of print].

[7] M.G. van Lier, A. Wagner, M.E. van Leerdam, et al., A review on the molecular diagnostics of Lynch syndrome: a central role for the pathology laboratory, J. Cell. Mol. Med. 14 (2010) 181-197, https://doi.org/10.1111/j.1582-4934.2009.00977.x Epub 2009 Nov 19.

[8] B.T. Niu, R.F.L. Hammond, S.L.S. Leen, C.B. Gilks, N. Singh, Two versus four immunostains for lynch syndrome screening in endometrial carcinoma,

Histopathology (2019), https://doi.org/10.1111/his.13898 [Epub ahead of print].

[9] M. Markow, W. Chen, W.L. Frankel, Immunohistochemical pitfalls: common mistakes in the evaluation of lynch syndrome surgical pathology clinics, Surg. Pathol. Clin. 10 (2017) 977-1007, https://doi.org/10.1016/j.path.2017.07.012.

[10] MLH1 Run 49, Nordic Immunohistochemical Quality Control, (2017) Last accessed May, 2019 https://www.nordiqc.org/downloads/assessments/86_81.pdf.

[11] B.T. Niu, R.F.L. Hammond, S.L.S. Leen, et al., Artefactual punctate MLH1 staining can lead to erroneous reporting of isolated PMS2 loss, Histopathology 73 (2018) 703-705, https://doi.org/10.1111/his.13663.

[12] M.B. Loughrey, P.D. Dunne, H.G. Coleman, S. McQuaid, J.A. James, Punctate MLH1 mismatch repair immunostaining in colorectal cancer, Histopathology 74 (2019) 795-797, https://doi.org/10.1111/his.13780.

[13] J.C. Watkins, M.R. Nucci, L.L. Ritterhouse, et al., Unusual mismatch repair immunohistochemical patterns in endometrial carcinoma, Am. J. Surg. Pathol. 40 (2016) 909-916, https://doi.org/10.1097/PAS.0000000000000663.

[14] O.M. Radu, M.N. Nikiforova, L.M. Farkas, A.M. Krasinskas, Challenging cases encountered in colorectal cancer screening for Lynch syndrome reveal novel findings: nucleolar MSH6 staining and impact of prior chemoradiation therapy, Hum. Pathol. 42 (2011) 1247-1258, https://doi.org/10.1016/j.humpath.2010.11.016.

[15] J. Shia, S. Holck, G. Depetris, J.K. Greenson, D.S. Klimstra, Lynch syndrome-associated neoplasms: a discussion on histopathology and immunohistochemistry, Fam. Cancer 12 (2013) 241-260, https://doi.org/10.1007/s10689-013-9612-4. 